\title{
Professores e maus-tratos - uma revisão teórica sobre reconhecimento, denúncia e capacitação
}

\author{
Teachers and child maltreatment - a theoretical review of \\ recognition, reports and capacity
Profesores y el abuso - una revisión teorica de reconocimiento, quejas y capacitación

\author{
JULIA DONAT* \\ TAYSE CONTER DE MOURA** \\ JANAÍNA DE CASTRO NUÑEZ CARVALHO**** \\ Christian HAAG KRISTENSEN****
}

\begin{abstract}
RESUMO
O presente trabalho resgata teoricamente o fenômeno nos maus-tratos na infância, contemplando principalmente a dificuldade ainda presente dos professores no reconhecimento de sintomas específicos e no encaminhamento adequado desses casos. Buscou-se compreender os principais conceitos de maus-tratos e de suas subdivisões (abuso sexual, abuso emocional, abuso físico e negligência), bem como as dificuldades que profissionais da educação encontram no manejo desses casos no dia a dia. Além disso, procurou-se verificar, a partir de uma revisão assistemática da literatura, programas de capacitação preventiva visando ao desenvolvimento profissional de professores e, por fim, enfatizar importantes aspectos que devem ser considerados nesse tipo de capacitação.
\end{abstract}

Palavras-chave: Abuso da criança. Crianças em idade escolar. Professores.

\begin{abstract}
This paper theoretically reviews the child maltreatment phenomenon, mostly considering the difficulty of teachers in the recognition of specific signs and symptoms and how to properly report the cases. We sought to understand the main concepts of abuse and their subdivisions (sexual abuse, emotional abuse, physical abuse and neglect) as well as the difficulties that teachers have with these cases on a daily bases. Furthermore, we aimed to verify through an unsystematic review of the literature prevention programs with teachers as the target audience, and finally, emphasize important aspects that must be considered for this type of training.

Keywords: Child abuse. School age children. Teachers.

\section{RESUMEN}

Este trabajo rescata teóricamente el fenómeno del maltrato infantil, especialmente teniendo en cuenta la dificultad que aún los maestros presentán en el reconocimiento de síntomas específicos y sus dudas en el manejo adecuado de estos casos. Hemos tratado de comprender los principales conceptos de abuso y sus subdivisiones (abuso sexual, abuso emocional, abuso físico y negligencia), así como las dificultades que los profesionales de la educación tienen en el manejo de estos casos. Además, tratamos de verificar a partir de una revisión de la literatura los programas de prevención con maestros, y, por último, destacamos los aspectos importantes que deben tenerse en cuenta en este tipo de formación.
\end{abstract}

Palabras clave: Abuso de niños. Niños em edad escolar. Profesores.

\footnotetext{
*É aluna de iniciação científica do grupo Cognição, Emoção e Comportamento, orientado pelo prof. Dr. Christian Haag Kristensen, do Programa de Pós-Graduação em Psicologia da Pontifícia Universidade Católica do Rio Grande do Sul (PRPPG-PUCRS). É também colaboradora do Núcleo de Estudos e Pesquisa em Trauma e Estresse (NEPTE-PUCRS).E-mail: <juliacdonat@gmail.com>.

** Acadêmica do 7o semestre de Psicologia da Pontifícia Universidade Católica do Rio Grande do Sul. Atualmente trabalha como Auxiliar de Pesquisa no Grupo Neuropsicologia Clínico Experimental do Pós-Graduação da Faculdade de Psicologia da PUCRS e realiza estágio ampliado no Núcleo de Estudo e Pesquisa sobre Trauma e Estresse na PUCRS. E-mail: <tay.conter@gmail.com>.

*** Mestra em Psicologia, área de concentração em Cognição Humana pela Pontifícia Universidade Católica do Rio Grande do Sul. Atualmente realiza Doutorado na área de Cognição Humana, no Núcleo de Estudos e Pesquisa em Trauma e Estresse (NEPTE-PUCRS).E-mail: <janainanunez@gmail.com>.

**** Doutor em Psicologia do Desenvolvimento pela Universidade Federal do Rio Grande do Sul. Professor Titular no Programa de Pós-Graduação em Psicologia na Pontifícia Universidade Católica do Rio Grande do Sul, Coordenador do Grupo de Pesquisa Cognição, Emoção e Comportamento e Coordenador Adjunto do Núcleo de Estudos e Pesquisa em Trauma e Estresse (NEPTE-PUCRS).
} 


\section{INTRODUÇÃO}

Os maus-tratos na infância são um importante problema de saúde em nível mundial. São definidos como cuidados físicos e emocionais inadequados, abuso sexual e/ou tratamento negligente que resultam em dano atual ou potencial à saúde, desenvolvimento ou dignidade de uma criança ou adolescente no contexto de um relacionamento de responsabilidade, confiança ou poder (KRUG et al., 2002; BUTCHART et al., 2006). A Organização Mundial da Saúde (OMS) estabeleceu quatro subtipos: abuso físico, abuso sexual, abuso emocional e negligência (WHO, 1990). Ainda que existam poucos estudos epidemiológicos no Brasil, estima-se que 10 a cada 1.000 crianças sejam vítimas de maus-tratos (TERRA et al., 2013) e que, a cada 20 casos, apenas um seja denunciado (MARTINS; MELLO-JORGE, 2010). Além disso, os maus-tratos representam uma das principais formas de morbidade que atinge a faixa etária de 5 a 19 anos no Brasil (JUNQUEIRA; DESLANDES, 2003).

As consequências dos maus-tratos incluem prejuízos à saúde mental e físicae ao desenvolvimento (BUTCHART et al., 2006). Estudos demonstraram relação entre maus-tratos e (1) alterações neurobiológicas, especialmente de estruturas do sistema límbico; (2) distúrbios psicopatológicos, como depressão, ansiedade, desregulação emocional, apego excessivo, dissociação, transtornos opositor e de conduta, transtorno de déficit de atenção e hiperatividade, abuso de álcool e outras drogas; (3) maior chance de desenvolver doenças físicas, como doenças cardiovasculares, de fígado, diabetes, obesidade, entre outras; e (4) alterações comportamentais e sociais, como agressividade, baixa autoestima, dificuldade de relacionamento, baixo rendimento escolar, capacidade cognitiva reduzida, entre outras (CICCHETTI; TOTH, 2005; PEREDA; GALLARDO-PUJOL, 2011; PIRES; MIAYAZAKI, 2005). Existem ainda consequências sociais importantes, tais como gastos judiciais e do sistema de saúde (PIRES; MIAYAZAKI, 2005).

Estudos revelam que $66,2 \%$ das vítimas de maustratos estão em idade escolar (HINES; BROWN, 2012). Professores, por terem contato direto, diário e prolongado, além de conhecerem os alunos sobre sua tutela, estão na "linha de frente" para a prevenção e reconhecimento de casos, já que podem, por exemplo, comparar comportamentos apresentados por potenciais vítimas com seus pares e também comportamentos atuais com aqueles apresentados anteriormente (ALBUHAIRAN et al., 2011; CRENSHAW; CRENSHAW; LICHTENBERG, 1995; NOGUEIRA, 1994). Além disso, os professores podem ser a única fonte de proteção, especialmente para crianças e adolescentes que têm familiares como agressores e não encontram a confiança e o apoio necessários à revelação da violência em mais ninguém (RISTUM, 2010). Em suma, os professores participam ativamente de grande parte do dia dessas crianças e, portanto, podem ter um papel de extrema importância na prevenção e no reconhecimento dos casos de maus-atos.

No Brasil, o Estatuto da Criança e do Adolescente (ECA) determina que professores devem comunicar à autoridade competente qualquer caso de seu conhecimento que envolva a suspeita ou a confirmação de maus-tratos, sob pena de pagamento de multa (BRASIL, 1990). Ainda existe um caminho a ser vencido entre o que diz o ECA e a prática profissional, uma vez que são quase inexistentes as notificações vindas de escolas, principalmente quando comparadas a outras fontes, como hospitais, vizinhos ou parentes de vítimas (RISTUM, 2010; VAGOSTELLO et al., 2003; SCHOLS; DE RUITER; ORY, 2013). A preparação desses profissionais para que sejam capazes de reconhecer os maus-tratos e atuar nesses casos é uma lacuna a ser preenchida tanto na literatura quanto na prática do dia a dia.

Reconhecendo-se o importante papel que a escola e os professores têm na identificação de casos de maus-tratos, e enfatizando que o papel do profissional não é investigar o caso, uma vez que essa é a função dos serviços de proteção à criança, o objetivo do presente artigo é fazer uma revisão teórica da literatura que procura entender as razões dos baixos índices de notificação por parte destes. Além disso, foi realizada uma busca sobre programas de capacitação para desenvolvimento profissional dos professores no tema dos maus-tratos já realizados nacionalmente e internacionalmente e discutido o que a literatura adverte que deve ser trabalhado nesse tipo de programa.

\section{RECONHECIMENTO E DENÚNCIA DE MAUS-TRATOS}

Pesquisas demonstram que os professores, em sua grande maioria, acreditam ter pouco conhecimento sobre maus-tratos e não são preparados em seus cursos de formação para reconhecer sinais e sintomas que possam auxiliar na identificação de possíveis vítimas (ALBUHAIRAN et al., 2011; CHOO et al., 2013; CRENSHAW; CRENSHAW; LICHTENBERG, 1995; KENNY, 2004; POTTER; BRIGGS; LUSHINGTON, 2002). Estudo realizado com professores norte-americanos demonstrou que, mesmo quando estes detectam casos de maus-tratos corretamente, não confiam suficientemente em sua avaliação e, por isso, acabam não realizando a denúncia (YANOWITZ; MONTE; TRIBBLE, 2003). Em uma amostra brasileira (VAGOSTELLO et al., 2003), os professores foram capazes de reconhecer sinais e sintomas em seus alunos, mas ainda assim não 
realizaram a denúncia por desconhecer informações adequadas sobre como e para quem denunciar. A falta de informação dos professores diante dos procedimentos de denúncia é um dado encontrado frequentemente em pesquisas internacionais (ABRAHAMS; CASEY; DARO, 1992; ALBUHAIRAN et al., 2011; CHOO et al., 2013; KENNY, 2001; KENNY, 2004; POTTER; BRIGGS; LUSHINGTON, 2002).

A união da dificuldade na identificação dos maustratos e o desconhecimento sobre os processos de denúncia são frequentemente apontados pela literatura como o maior impeditivo para que esses casos sejam denunciados para os órgãos competentes (ABRAHAMS; CASEY; DARO, 1992; BECK; OGLOFF; CORBISHLEY, 1994; KENNY, 2001). Professores com conhecimento sobre maus-tratos e planos sobre como realizar a denúncia têm quase duas vezes mais chance de efetivamente realizála (GOEBBELS et al., 2008). Ainda que existam outras questões que possam dificultar a decisão de denunciar, a falta de familiaridade com o tema parece ser a mais importante delas, uma vez que as pesquisas também trazem evidências de que professores que já denunciaram anteriormente têm maior chance de realizar uma nova denúncia (GOEBBLES et al., 2008; KENNY, 2004; WALSH et al., 2012; WEBSTER et al., 2005).

Existem outras características diretamente relacionadas aos professores que podem ser empecilhos para a denúncia: acreditar que esta trará consequências negativas para a criança (ABRAHAMS; CASEY; DARO, 1992; BECK; OGLOFF; CORBISHLEY, 1994; SCHOLS; DE RUITER; ORY, 2013; WEBSTER et al., 2005); falta de confiança nos serviços de proteção à criança (BECK; OGLOFF; CORBISHLEY, 1994; TITE, 1994); receio de realizar uma falsa denúncia (BECK; OGLOFF; CORBISHLEY, 1994; TITE, 1994; SCHOLS; DE RUITER; ORY, 2013); além do medo de repercussões negativas com os pais (e.g., retaliação, processo legal) (ABRAHAMS; CASEY; DARO, 1992; KENNY, 2004; SCHOLS; DE RUITER; ORY, 2013). Há ainda evidências de que professores com mais anos de experiência (GOEBBLES et al., 2008; KENNY, 2001), com maior nível educacional (GOEBBLES et al., 2008; WEBSTER et al., 2005) e do sexo feminino (CRENSHAW; CRENSHAW; LICHTENBERG, 1995; FINALYSON; KOOCHER, 1991; KENNY, 2001) têm maiores chances de denunciar casos de maus-tratos.

Mesmo que as pesquisas até hoje realizadas tenham apresentado como fator relevante para o baixo índice de denúncias o pouco conhecimento dos professores sobre sinais e sintomas de maus-tratos e sobre procedimentos práticos em como realizar a denúncia, apenas esses dados não são claros. Na verdade, são imprecisos em relação à ideia de que programas de desenvolvimento profissional ligados a maus-tratos aumentariam o índice de denúncias, sendo fundamental a realização de pesquisas sobre intervenções (WALSH et al., 2008). No entanto, é imprescindível ressalvar que existem evidências de que professores mais familiarizados com sinais de maustratos tiveram maior tempo de formação relacionado ao tema (FENG et al., 2012; MCINTYRE, 1987), ainda que a maioria tenha informado nunca ter participado de qualquer programa de capacitação para reconhecimento de maus-tratos e/ou ter considerado este inadequado ou insuficiente (ABRAHAMS; CASEY; DARO, 1992; GRANVILLE-GARCIA et al., 2009; KENNY, 2001; SCHOLS; DE RUITER; ORY, 2013; LEVIN, 1983; MCINTYRE, 1987). É importante ressaltar, também, que grande parte dos professores reconhece a importância de sua função na prevenção de maus-tratos e demonstra interesse em aprender mais sobre esse tema (MCINTYRE, 1987; POTTER; BRIGGS; LUSHINGTON, 2002).

Podem-se apontar múltiplas falhas para que se tenha hoje a baixa notificação por parte de escolas e seus professores em casos de maus-tratos: o tema não é trabalhado na formação dos professores; as escolas não incentivam os professores a se capacitarem, seja pela não oferta de cursos ou programas de desenvolvimento profissional ou até mesmo deixando de apoiá-los em alguns casos de denúncia. Outra falha importante é a falta de um trabalho interdisciplinar com áreas como a psicologia ou a psicopedagogia, mais familiarizadas com o tema, que poderiam auxiliar os professores e as escolas não apenas no reconhecimento dos sinais e sintomas, mas também no apoio do processo de realização das denúncias.

\section{EXEMPLOS DE PROGRAMAS DE CAPACITAÇÃO PREVENTIVA EM MAUS-TRATOS PARA PROFESSORES}

O maior objetivo dos programas de prevenção relacionados a maus-tratos é a diminuição da incidência de situações de abuso (BOLEN, 2003). Ainda que se reconheça a situação privilegiada em que os professores estão, por sua convivência direta com as crianças e adolescentes, no combate às situações de maus-tratos, são escassos os estudos que investiguem empiricamente os resultados de programas de capacitação preventivos sobre o tema para essa população-alvo. Por exemplo, programas de prevenção com modelos de visita domiciliar são mais corriqueiros do que aqueles em meio escolar (MIKTON; BUTCHART, 2009). Encontrados mais facilmente, dois programas de prevenção de nível secundário, que têm como finalidade a detecção precoce de sintomatologia e de problemas sociais (ALMEIDA, 2005), foram aqui descritos. Além disso, foi descrito um programa norteamericano de prevenção de nível terciário que, além do 
tratamento, procura prevenir a recorrência da situação de violência identificada (ALGERI; SOUZA, 2006). Foi constatado, durante a realização da revisão sobre o assunto, que os programas de capacitação para desenvolvimento profissional dos professores no tema dos maus-tratos utilizam-se de diferentes métodos e instrumentos de análise de resultados, não havendo uma padronização internacional na realização de tal capacitação.

Na Europa, Cerezo e Pons-Salvador (2004) realizaram uma intervenção nas Ilhas Baleares (uma das comunidades autônomas da Espanha) com mais de 180 profissionais de agências de saúde e serviços sociais, além de 251 profissionais da educação (e.g., professores de séries diversificadas, psicólogos e pedagogos). Estes foram instigados a realizar uma detecção mais adequada dos sinais de maus-tratos, através de oficinas de 16 a 20 horas, com objetivos relacionados à promoção de conhecimentos gerais sobre o assunto, sensibilização para realização da denúncia e procedimentos adequados para notificações. Técnicas como dinâmica de grupo, escuta de experiências, medos e preocupações dos participantes e incentivo à mudança de atitudes foram utilizadas, além de serem disponibilizadas linhas telefônicas para esclarecimentos após a capacitação. Como resultado, as detecções foram triplicadas.

O programa desenvolveu também uma folha de comunicação ao serviço competente para realizar a investigação, com o objetivo de facilitar o encaminhamento de casos suspeitos. Esse instrumento é composto por três seções que incluem questões sobre: (1) sinais e sintomas de maus-tratos; (2) questões familiares e circunstâncias do lar em que a criança vive; e (3) quem é a pessoa que poderia ser a responsável pelo dano à criança. $\mathrm{O}$ instrumento mostrou resultados positivos, já que proporcionou a simplificação da denúncia e o estreitamento do contato entre as escolas e as instituições responsáveis. Esse estudo demonstrou que é possível melhorar o sistema de detecção de maus-tratos com programas que incluam o desenvolvimento e a capacitação de vários profissionais, além de demonstrar que a escola possui um papel único e relevante na constatação de maus-tratos, sendo responsável pelo maior número de detecções.

No Brasil, Brino e Williams (2008) realizaram uma intervenção cujo foco foi a capacitação de professores como agentes de prevenção, especificamente do abuso sexual infantil, que contou com a participação de 100 professores da Educação Infantil. Estes participaram de 12 encontros semanais pelo período de três meses, divididos em partes teórica e prática. A parte teórica englobou conceitos como a definição de abuso sexual infantil, reconhecimento de sintomas e ações preventivas para professores; enquanto a parte prática incluiu oficinas para os familiares das crianças sobre prevenção de abuso sexual e, aos alunos, oficinas sobre habilidades de autoproteção. Através do instrumento de pesquisa desenvolvido pelos autores (e.g., Exercício de Abuso Sexual para Professores) e da escala Indicadores de Abuso Sexual (IAS) (FLORES et al., 2001), foram encontradas evidências de que, ao longo da capacitação, os professores melhoraram no reconhecimento dos sintomas específicos do abuso sexual (e.g., curiosidade sexual excessiva, autoconceito negativo e sentimentos de vergonha e/ou culpa). Mas não se encontrou o mesmo resultado em relação aos sintomas inespecíficos (e.g., transtornos alimentares e pesadelos). Nesse sentido, foi sugerido o acompanhamento dos professores na decisão da denúncia por parte das escolas. O bom desempenho destes no instrumento Exercício sobre Abuso Sexual para Professores indica maior capacidade na descrição dos procedimentos para denúncia, o que, embora não garanta a atuação adequada em casos do dia a dia, pode servir como um primeiro passo para que as denúncias sejam feitas.

Com menos programas descritos na literatura, a prevenção terciária contempla a intervenção e a gestão do problema (FOWLER; GRAY, 1983), ou seja, busca trabalhar com crianças que estão com questões importantes circunscritas aos maus-tratos sofridos, o que também evita reincidências (ALGERI; SOUZA, 2006). Sabendo-se que um dos sintomas de crianças que passaram por situações adversas de maus-tratos é a queda no rendimento escolar (BEARD, 2014), torna-se importante a intervenção também nesse sentido. Um projeto piloto intitulado de Programa de Sucesso Escolar (MALLET, 2012), em Ohio, atuou em 18 escolas públicas, por 24 meses, e notou melhora significativa no desempenho de alunos vítimas de maus-tratos em construtos considerados importantes no desempenho escolar (e.g., capacidade intelectual geral, linguagem, leitura e compreensão, escrita básica e raciocínio e cálculos matemáticos). O programa, que considerava importante o trabalho colaborativo entre escola, família e Agências de Serviços da Criança, iniciou-se em 2001 e ganhou força ao longo dos anos, tendo atingido, em 2009, 615 crianças com questões de abuso ou negligência.

O programa contava com um orientador que trabalhava em conjunto com a criança, com a família e com o professor de sala de aula, estimulando o trabalho em equipe.Ele se reunia com a criança durante a semana (de uma a quatro horas, dependendo da necessidade), na residência da criança, e se concentrava em dificuldades relacionadas ao aprendizado ou a quaisquer questões que pudessem estar dificultando a evolução esperada do aluno em sala de aula. Também foram disponibilizados computadores com softwares educacionais que visavam ao treinamento de organização diária para manter o interesse 
da criança nas atividades propostas. Os resultados foram bastante promissores, uma vez que os participantes melhoraram seu rendimento duas vezes mais rápido do que a média nacional, ou seja, do que seria esperado para sua idade.

\section{ELABORAÇÃo DE UM PROGRAMA DE CAPACITAÇÃO PREVENTIVA EM MAUS-TRATOS}

A literatura assegura que, na realização de capacitações cujo foco sejam os maus-tratos, a adoção de medidas empiricamente comprovadas devem ser consideradas, principalmente para que o bem-estar da criança seja efetivamente reassegurado (ALVAREZ et al., 2004; TOTH; MANLY, 2011). Ainda que, como citado anteriormente, sejam escassos os estudos específicos sobre programas de capacitação preventiva relacionados a maus-tratos, as orientações aqui descritas são baseadas em recomendações gerais da literatura específica da área que visam à prevenção de situações de maus-tratos com diferentes públicos-alvo.

Inicialmente, espera-se que o programa seja adequadamente formulado de acordo com a legislação vigente, considerando-se a obrigatoriedade da notificação, a maneira de ser realizada e a forma de responsabilização de quem não a fizer (ALVAREZ et al., 2004). Torna-se fundamental que se opere de acordo com as demandas do contexto no qual será realizado, para que, de fato, as crianças vítimas de quaisquer abusos sejam protegidas (TOTH; MANLY, 2011). A falta de programas de desenvolvimento profissional bem estruturados e embasados no contexto em que a violência ocorre, além de gerar subnotificação, pode, ao contrário, causar efeito de generalização de denúncias e colocar muitas famílias, já em situações de vulnerabilidade, em processos de investigações e estresse desnecessários (KRASE, 2013). No Brasil, além da notificação ser obrigatória por lei (BRASIL, 1990), existe uma exposição frequente das crianças à violência de maneira geral. É importante que, durante a capacitação profissional, os professores estejam conscientes de tais particularidades de nosso país.

Uma vez que pesquisas demonstram que professores podem apresentar insegurança diante de falsas denúncias (BECK; OGLOFF; CORBISHLEY, 1994; TITE, 1994; SCHOLS; DE RUITER; ORY, 2013), é fundamental que o programa contemple informações que reforcem que denúncias devem ser realizadas de acordo com as suspeitas que tiverem (BURNS; LAKE, 1983; KALICHMAN, 1999), eximindo-os da responsabilidade de ganhar informações sobre o caso antes do relato (KENNY, 1998). E que abranja, também, os procedimentos específicos para realização da denúncia de forma adequada (ALVAREZ et al, 2004). É de grande importância, ainda, que os professores compreendam que seu papel não engloba a investigação do caso, sendo esta a função dos serviços de proteção à criança, e que eles estejam familiarizados sobre questões práticas da denúncia, tais como onde e para quem realizá-la.

Para maior compreensão do que são maus-tratos, acrescenta-se que todo o programa de capacitação seja conduzido a partir da subdivisão desse termo, ou seja, pelas quatro categorias que o representam (i.e., abuso físico, abuso sexual, abuso emocional e negligência) (ALVAREZ et al., 2004). Também se sugere que, ao longo da programação, sejam incluídos exercícios em que vinhetas sejam lidas e classificadas adequadamente conforme subtipos de maus-tratos (ALVAREZ et al., 2004). Programas de foco mais empírico recomendam o treinamento de habilidades através de role-plays e feedbacks (DONOHUE et al., 2002), para que o profissional aprenda como transmitir informações difíceis de relatar (ALEXANDER, 1990).

A literatura aponta para a importância do envolvimento familiar da vítima no processo da denúncia, propiciando clima de maior colaboração (BROMLEY; RIOLO, 1988) e apoio para resolução de problemas, ao invés de atitudes punitivas (ALGERI; SOUZA, 2006). Além disso, programas de capacitação devem contemplar a sensibilização no manejo com a criança, para que os participantes expressem preocupação de forma adequada e expliquem com cuidado o processo de investigação que se dará a partir da denúncia (ALVAREZ et al, 2004; BRINO; WILLIAMS, 2008). Para assegurar a qualidade de treinamentos ao longo do tempo, grupos e supervisões podem ser oferecidos, justamente por criarem compartilhamento de experiências, frustrações e ansiedades (ALGERI; SOUZA, 2006; TOTH; MAINLY, 2011).

É indispensável que os professores sejam informados sobre os sinais e sintomas de maus-tratos mais comumente apresentados pelas crianças, com a ressalva de que a apresentação é muito variada caso a caso. Os sintomas podem ser divididos em: (1) internalizantes (e.g., sentimentos de estar sozinho ou isolado das figuras afetivas, embotamento afetivo, interesse diminuído em atividades habituais, pesadelos, queixas psicossomáticas, sensação de futuro abreviada); e (2) externalizantes (e.g., transtornos de sono, irritabilidade, raiva, hipervigilância, resposta de sobressalto exagerada) (APA, 2013; COATES; GAENSBAUER, 2009; EISENSTEIN; JORGE; LIMA, 2009; KAMINER; SEEDAT; STEIN, 2005). Além disso, crianças mais novas podem apresentar problemas de aprendizagem, dificuldade de concentração e tendência a manifestar novos medos em relação a incidentes específicos (COOK-COTTONE, 2004; PYNOOS et al., 2009). 
Os adolescentes, por sua vez, manifestam reações semelhantes às de adultos, podendo apresentar sentimentos de futuro abreviado, sintomas de depressão e engajamento a comportamentos de risco (DYREGROV; YULE, 2006; PYNOOS et al., 2009).

\section{CONSIDERAÇÕES FINAIS}

O número de casos de crianças vítimas de maus-tratos no Brasil é preocupante, principalmente quando apenas uma ínfima parte destes é denunciada. As consequências dos maus-tratos acarretam prejuízos que podem se apresentar tanto no curto quanto no longo prazo, na vida da vítima. Programas de prevenção se tornam extremamente indicados, pois objetivam justamente à diminuição desses altos índices (BOLEN, 2003).

Ainda que os professores possuam contato diário com possíveis vítimas e, portanto, grande oportunidade para o reconhecimento de seus sintomas, esses não são frequentemente instrumentalizados para a realização de denúncias, tanto por falta de capacitação sobre maustratos durante a sua formação quanto pela falta de apoio e oportunidades de desenvolvimento profissional que recebem das escolas em que trabalham para lidar com o tema. São escassos os estudos sobre programas de capacitação preventivos específicos com essa população. E mesmo que a literatura reconheça as dificuldades dos professores, tanto no reconhecimento de sinais e sintomas quanto no procedimento adequado para realizar a denúncia, parecem ser pequenos os esforços para a construção de conhecimento científico sobre as maneiras mais eficazes de realizar tal tipo de capacitação.

Ao serem revisados programas já realizados internacionalmente, encontram-se evidências de que os professores adquirem capacidade de detecção de maustratos e de formas de encaminhamento após cursos de desenvolvimento profissional adequados. Além disso, programas focados em trabalhar não apenas no reconhecimento de sinais e sintomas, mas também em como realizar a denúncia de maus-tratos, parecem ser mais bem-sucedidos. Recomenda-se, para que os professores adquiram maior habilidade em detectar sinais de maustratos e realizar processos de denúncia, que eles sejam assistidos pelas escolas, de forma a sentirem-se amparados na tomada de sua decisão em realizar a denúncia.

Até o momento, os programas de prevenção têm apresentado evidências preliminares positivas. No entanto, são necessários mais estudos empíricos sobre a efetividade de tais programas, para que se tenham evidências mais sólidas sobre esse tipo de capacitação. No entanto, pode-se considerar que os estudos já realizados até o momento parecem ter dado um primeiro passo para a realização de uma denúncia adequada por parte de educadores, pois propiciam intervenções precoces que podem evitar reincidências de situações adversas, garantindo à criança o cuidado e a proteção que, constitucionalmente, são de seu direito.

\section{REFERÊNCIAS}

ABRAHAMS, N.; CASEY, K.; DARO, D. Teachers'knowledge, attitudes, and beliefs about child abuse and its prevention. Child abuse $\&$ neglect, v. 16 , n. 2, p. 229-238, 1992. http://dx.doi. org/10.1016/0145-2134(92)90030-U

ALBUHAIRAN, F. S. et al. Self reported awareness of child maltreatment among school professionals in Saudi Arabia: Impact of $\mathrm{CRC}$ ratification. Child abuse \& neglect, v. 35, n. 12, p. 1032-1036, 2011. http://dx.doi.org/10.1016/j. chiabu.2011.10.002

ALGERI, S.; SOUZA, L. M. Violence against children and adolescents: a challenge in the daily work of the nursing team. Revista Latino-Americana de Enfermagem, v. 14, n. 4, p. 625-631, 2006. http://dx.doi.org/10.1590/S010411692006000400023

ALMEIDA, L. M. Da prevenção primordial à prevenção quaternária. Revista Portuguesa de Saúde Pública, v. 23, n. 1, 91-96, 2005.

ALVAREZ, K. M. et al. Why are professionals failing to initiate mandated reports of child maltreatment, and are there any empirically based training programs to assist professionals in the reporting process? Aggression and Violent Behavior, v. 9, n. 5, p. 563-578, 2004. http://dx.doi.org/10.1016/j.avb.2003. 07.001

AMERICAN PSYCHIATRIC ASSOCIATION et al. Diagnostic and statistical manual of mental disorders (DSM). Washington, DC: American Psychiatric Association, 2013. p. 143-147.

BEARD, K. W.; Wolf, E. M. Modification in the proposed diagnostic criteria for Internet addiction. CyberPsychology \& Behavior, v. 4, n. 3, p. 377-383, 2001. http://dx.doi. org/10.1089/109493101300210286

BECK, Kirk A.; OGLOFF, James R. P.; CORBISHLEY, Anne. Knowledge, compliance, and attitudes of teachers toward mandatory child abuse reporting in British Columbia. Canadian Journal of Education/Revue/Canadienne de l'Education, p. $15-29,1994$.

BOLEN, R. M. Child sexual abuse: prevention or promotion? Social Work, v. 48, n. 2, p. 174-185, 2003.

BRASIL. Estatuto da Criança e do Adolescente, 1990.

BRINO, R.; WILLIAMS, L. C. Professores como agentes de prevenção do abuso sexual infantil. Educação \& Realidade, v. 33, n. 2, p. 209-229, 2008.

BROMLEY, M. A.; RIOLO, J. Complying with mandated child protective reporting: a challenge for treatment professionals. Alcoholism Treatment Quarterly, v. 5, n. 3-4, p. 83-96, 1989. http://dx.doi.org/10.1300/J020V05N03_07

BURNS, G. E.; LAKE, D. E. A sociological perspective on implementing child abuse legislation in education. Interchange, v. 14, n. 2 , p. $33-53$, 1983. http://dx.doi.org/10.1007/BF 01808080 
BUTCHART, A. et al. Preventing child maltreatment: a guide to taking action and generating evidence. World Health Organization, 1992.

CEREZO, M. A.; Pons-Salvador, G. Improving child maltreatment detection systems: a large-scale case study involving health, social services, and school professionals. Child Abuse \& Neglect, v. 28, n. 11, p. 1153-1169, 2004. http:// dx.doi.org/10.1016/j.chiabu.2004.06.007

CHOO, W. Y. et al. Are Malaysian teachers ready to assume the duties of reporting child abuse and neglect? Child Abuse Review, v. 22, n. 2, p. 93-107, 2013. http://dx.doi.org/10.1002/ car.2241

CICCHETTI, D.; TOTH, S. L. Child maltreatment. Annual Review Clinical Psychology, v. 1, p. 409-438, 2005. http:// dx.doi.org/10.1146/annurev.clinpsy.1.102803.144029

COATES, S.; GAENSBAUER, T. J. Event trauma in early childhood: symptoms, assessment, intervention. Child and Adolescent Psychiatric Clinics of North America, v. 18, n. 3, p. 611-626, 2009. http://dx.doi.org/10.1016/j. chc.2009.03.005

COOK-COTTONE, C. Childhood posttraumatic stress disorder: diagnosis, treatment, and school reintegration. School Psychology Review, v. 33, n. 1, p. 127-139, 2004.

CRENSHAW, W. B.; CRENSHAW, L. M.; LICHTENBERG, James W. When educators confront child abuse: an analysis of the decision to report. Child Abuse \& Neglect, v. 19, n. 9, p. 1095-1113, 1995. http://dx.doi.org/10.1016/0145-2134(95) $00071-\mathrm{F}$

DONOHUE, B. et al. A standardized method of diplomatically and effectively reporting child abuse to state authorities: a controlled evaluation. Behavior Modification, v. 26, n. 5, p. 684-699, 2002. http://dx.doi.org/10.1177/014544502236657

DYREGROV, A.; YULE, W. A review of PTSD in children. Child and Adolescent Mental Health, v. 11, n. 4, p. 176-184, 2006. http://dx.doi.org/10.1111/j.1475-3588.2005.00384.x

EISENSTEIN, E.; JORGE, E.; LIMA, L. A. Transtorno do estresse pós-traumático e suas repercussões clínicas durante a adolescência. Revista Adolescência \& Saúde, v. 6, n. 3, 2009.

FENG, J. et al. Contextual effects on kindergarten teachers' intention to report child abuse. Journal of Community Psychology, v. 40, n. 7, p. 886-890, 2012. http://dx.doi. org/10.1002/jcop. 21508

FINLAYSON, L. M.; KOOCHER, G. P. Professional judgment and child abuse reporting in sexual abuse cases. Professional Psychology: Research and Practice, v. 22, n. 6, p. 464, 1991. http://dx.doi.org/10.1037/0735-7028.22.6.464

FLORES, R. Z. et al. O efeito da capacitação em profissionais da area da saúde no reconhecimento dos indicadores de abuso sexual em crianças e adolescentes. In: REUNIÃO ANUAL DE PSICOLOGIA, XXXI., 2001, Rio de Janeiro. Anais... (resumos). Rio de Janeiro: Sociedade Brasileira de Psicologia, 2001. p. 274-274.

FOWLER, G.; GRAY, M. Opportunities for prevention in general practice. In: GRAY, M.; FOWLER, G. (Org.). Preventive medicine in general practice. Oxford: Oxford University Press, 1983.
GOEBBELS, A. F. G. et al. Teachers' reporting of suspected child abuse and neglect: behavior and determinants. Health Education Research, v. 23, n. 6, p. 941-951, 2008. http://dx.doi. org/10.1093/her/cyn030

GRANVILLE-GARCIA, A.; SILVA, M.; DE MENEZES, V. Abuse of children and adolescent: a study in the city of São Bento do Una, PE, Brazil. Brazilian Research in Pediatric Dentistry and Integrated Clinic, v. 8, n. 3, p. 301-307, 2009.

HINES, L.; BROWN, R. Child abuse awareness and reporting in schools. NASN School Nurse, v. 27, n. 3, p. 122-124, 2012. http://dx.doi.org/10.1177/1942602X12440287

JUNQUEIRA, M. et al. Resiliência e maus-tratos à criança. Cadernos de Saúde Pública, v. 19, n. 1, p. 227-235, 2003. http:// dx.doi.org/10.1590/S0102-311X2003000100025

KALICHMAN, S. Reported cases of suspected child abuse. American Psychological Association, 1999. http://dx.doi. org/10.1037/10337-006

KAMINER, D.; SEEDAT, S.; Stein, D. J. Post-traumatic stress disorder in children. World Psychiatry, v. 4, n. 2, p. 121, 2005.

KENNY, M. C. Child abuse reporting: teachers' perceived deterrents. Child Abuse \& Neglect, v. 25, n. 1, p. 81-92, 2001. http://dx.doi.org/10.1016/S0145-2134(00)00218-0

KENNY, M. C. Teachers' attitudes toward and knowledge of child maltreatment. Child Abuse \& Neglect, v. 28, n. 12, p. 13111319, 2004. http://dx.doi.org/10.1016/j.chiabu.2004.06.010

KRASE, K. S. Educational personnel as reporters of suspected child maltreatment. Children \& Schools, v. 35, n. 3, p. 147-154, 2013. http://dx.doi.org/10.1093/cs/cdt008

KRUG, E. G. et al. The world report on violence and health. The Lancet, v. 360, n. 9339, p. 1083-1088, 2002.

LEVIN, P. G. Teachers' perceptions, attitudes, and reporting of child abuse/neglect. Child Welfare: Journal of Policy, Practice, and Program, 1983.

MALLETT, C. A. The school success program: improving maltreated children's academic and school-related outcomes. Children \& Schools, v. 34, n. 1, p. 13-26, 2012. http://dx.doi. org/10.1093/cs/cdr004

MARTINS, C.; DE MELLO JORGE, M. Abuso sexual na infância e adolescência: perfil das vítimas e agressores em município do sul do Brasil. Texto \& Contexto Enfermagem, v. 19 , n. 2, p. 246-255, 2010. http://dx.doi.org/10.1590/S010407072010000200005

MCINTYRE, T. C. Teacher awareness of child abuse and neglect. Child Abuse \& Neglect, v. 11, n. 1, p. 133-135, 1987. http://dx.doi.org/10.1016/0145-2134(87)90042-1

MIKTON, C.; BUTCHART, A. Child maltreatment prevention: a systematic review of reviews. Bulletin of the World Health Organization, v. 87 , n. 5, p. 353-361, 2009. http://dx.doi. org/10.2471/BLT.08.057075

NOGUEIRA, M. A. O papel da escola na prevenção dos maus-tratos na infância e adolescência. Rev. Bras. Saúde Esc., v. 3, n. 1/4, p. 241-3, 1994.

PEREDA, N.; GALLARDO-PUJOL, D. Revisión sistemática de las consecuencias neurobiológicas del abuso sexual infantil. Gaceta Sanitaria, v. 25, n. 3, p. 233-239, 2011. http://dx.doi. org/10.1016/j.gaceta.2010.12.004 
PIRES, A.; MIYAZAKI, M. C. O. S. Maus-tratos contra crianças e adolescentes: revisão da literatura para profissionais da saúde. Arquivos de Ciências da Saúde, v. 12, n. 1, p. 42-49, 2005.

POTTER, G.; BRIGGS, F.; LUSHINGTON, K. What Singaporean teachers and social workers say about child abuse: facts versus myths. Teaching and Learning, v. 23, n. 1, p. 13-25, 2002.

PYNOOS, R. S. et al. DSM-V PTSD diagnostic criteria for children and adolescents: A developmental perspective and recommendations. Journal of Traumatic Stress, v. 22, n. 5, p. 391-398, 2009. http://dx.doi.org/10.1002/jts.20450

RISTUM, M. A violência doméstica contra crianças e as implicações da escola: school implications. Temas em Psicologia, v. 18, n. 1, p. 231-242, 2010.

SCHOLS, M.; DE RUITER, C.; ÖRY, F. G. How do public child healthcare professionals and primary school teachers identify and handle child abuse cases? A qualitative study. BMC Public Health, v. 13, n. 1, p. 807, 2013. http://dx.doi. org/10.1186/1471-2458-13-807

TERRA, B. et al. Maus-tratos infantis. Revisão da literatura. Revista Brasileira de Ortopedia, v. 48, n. 1, p. 11-16, 2013. http://dx.doi.org/10.1016/j.rbo.2012.03.003

TITE, R. Detecting the symptoms of child abuse: classroom complications. Canadian Journal of Education/Revue Canadienne de l'Education, p. 1-14, 1994.
TOTH, S. L.; MANLY, J. T. Bridging research and practice: challenges and successes in implementing evidence-based preventive intervention strategies for child maltreatment. Child Abuse \& Neglect, v. 35, n. 8, p. 633-636, 2011. http://dx.doi. org/10.1016/j.chiabu.2011.05.003

VAGOSTELLO, L. et al. Violência doméstica e escola: um estudo em escolas públicas de São Paulo. Paidéia (Ribeirão Preto), v. 13, n. 26, p. 191-196, 2003. http://dx.doi.org/10.1590/ S0103-863X2003000300008

WALSH, K. et al. Understanding teachers' reporting of child sexual abuse: Measurement methods matter. Children and Youth Services Review, v. 34, n. 9, p. 1937-1946, 2012. http:// dx.doi.org/10.1016/j.childyouth.2012.06.004

WEBSTER, S. W. et al. Overreporting and underreporting of child abuse: teachers' use of professional discretion. Child Abuse \& Neglect, v. 29, n. 11, p. 1281-1296, 2005. http:// dx.doi.org/10.1016/j.chiabu.2004.02.007

YANOWITZ, Karen L.; MONTE, Emmanuelle; TRIBBLE, Jamie R. Teachers' beliefs about the effects of child abuse. Child Abuse \& Neglect, v. 27, n. 5, p. 483-488, 2003. http:// dx.doi.org/10.1016/S0145-2134(03)00033-4

Submetido em: 19/04/2015

Aprovado em: 15/12/2015 